\title{
Calibration of the Tully-Fisher relation in the field
}

\author{
S. Rauzy \\ ANPE and Centre de Physique Théorique, CNRS, Luminy Case 907, F-13288 Marseille Cedex 9, France
}

Received May 3; accepted December 20, 1996

\begin{abstract}
A new technique for calibrating slope and relative zero-point of Tully-Fisher like relations by using a sample of distant field galaxies is proposed. Based on a null-correlation approach (NCA), the technique is insensitive to the presence of selection effects on apparent magnitude $m$ and on log line-width distance indicator $p$. This interesting property is used for discarding nearby galaxies of the observed sample. It is shown that such a subsampling allows in effect to attenuate biases on calibration parameters created by the presence of radial peculiar velocities.
\end{abstract}

Key words: galaxies: distances and redshifts — distance scale

\section{Introduction}

During this last decade, increasing efforts have been made attempting to extract information concerning the distribution of mass in the universe from the radial peculiar velocity field of distant galaxies (see for example Aaronson et al. 1986; Lynden-Bell et al. 1988; Bertschinger et al. 1990; Rauzy et al. 1992; Newsam et al. 1995; Rauzy et al. 1995). Quantitative results based on peculiar velocity studies, such as constraints on the value of the density parameter $\Omega_{0}$, can nowadays be found in the literature (see Dekel 1994 for a review). Reliability of such results is however closely related to the various working hypotheses assumed throughout the successive steps of the analysis.

The first of these steps is to obtain redshiftindependent estimates of galaxies distance. It is performed by using Tully-Fisher (TF) like relations (Tully-Fisher 1977 for spirals and Faber-Jackson 1976 for ellipticals). These observed statistical relations linearly correlate the absolute magnitude $M$ (or similar quantity) of a galaxy with an observable parameter $p\left(p=\log V_{\text {rot }}\right.$ for spirals and $p=\log \sigma$ for ellipticals). Assuming that the TF relation has been correctly calibrated, an estimate of the

Send offprint requests to: S. Rauzy (rauzy@cpt.univ-mrs.fr) absolute magnitude $M$ is obtained by measuring the observable $p$. Measurement of the apparent magnitude $m$ (or similar quantity) provides then with an estimate of the distance of the galaxy, and comparing it with the redshift $z$ finally furnishes the deviation from the mean Hubble flow (i.e. the radial peculiar velocity).

The preliminar calibration step of the TF relation is of crucial importance for kinematical analyses since errors on the calibration parameters interpret indeed as fictitious large-scale and coherent peculiar velocity fields. Unfortunatly, selection effects in observation, such as upper bound in apparent magnitude, bias the estimates of the TF calibration parameters. Many studies devoted to correct on these biases have been already proposed (see for example Schechter 1980; Bottinelli et al. 1986; LyndenBell et al. 1988; Fouqué et al. 1990; Hendry \& Simmons 1990; Teerikorpi 1990; Bicknell 1992; Triay et al. 1994; Willick 1994; Hendry \& Simmons 1994; Sandage 1994; Willick et al. 1995; Willick et al. 1996; Rauzy \& Triay 1996; Ekholm 1996; Triay et al. 1996).

Motivations leading to introduce a new calibration technique are twofold. First, bias correction requires generally a full description of the calibration sample (i.e. the specific shapes of the observational selection function on $m$ and $p$ and of the luminosity function have to be assumed). Since available samples are often constituted of data inherited from various observational programs, modelization of such characteristics still remains a difficult problem. The philosophy is herein to reduce as far as possible the number of dubious assumptions made on these composite samples when deriving calibration parameters. Second, it is not clear how existing calibration procedures are affected by the presence of radial peculiar velocities. The aim is herein to quantify, and if possible to minimize, influences of peculiar velocity fields on the estimates of the calibration parameters.

In Sect. 2 is summarized the basic statistical model describing Tully-Fisher like relations. The new calibration technique is presented Sect. 3. Cumbersome calculations and proofs can be found Appendices A, B and C. Potentialities of the method are illustrated in Appendix 
D where NCA calibration of the Mathewson spirals field galaxies sample is performed.

\section{Distance and velocity estimates}

The Tully-Fisher like relations are based on an observed linear correlation between the absolute magnitude $M$ (or similar quantity such as $-5 \log _{10} D$ with $D$ the linear diameter) and the log line-width distance indicator $p$ of galaxies $\left(p \approx \log V_{\text {rot }}\right.$ for spirals and $p \approx \log \sigma$ for ellipticals). They allow to estimate distance and radial peculiar velocity of an individual galaxy from its measured apparent magnitude $m$ (or similar quantity such as $-5 \log _{10} d$ with $d$ the apparent diameter), parameter $p$ and redshift $z$. In this section, we recall in mind the basic statistical model describing these relations (see Triay et al. 1994 or Rauzy \& Triay 1996 for details).

Regardless of the distance of the galaxy, selection effects in observation and measurement errors, the theoretical probability density $(p d)$ in the $M-p$ plane reads:

$\mathrm{d} P_{1}=F(M, p) \mathrm{d} M \mathrm{~d} p$.

The Direct (i.e. Forward) Tully-Fisher (DTF) relation assumes that it exists a random variable $\zeta^{D}$,

$\zeta^{D}=\widetilde{M}(p)-M=a^{D} p+b^{D}-M$

statistically independent of $p$ such as the theoretical $p d$ of Eq. (1) rewrites:

$\mathrm{d} P_{1}=f_{p}(p) \mathrm{d} p g\left(\zeta^{D} ; 0, \sigma_{\zeta}^{D}\right) \mathrm{d} \zeta^{D}$

where $f_{p}(p)$ is the distribution function of the variable $p$ in the $M-p$ plane. The random variable $\zeta^{D}$ of zero mean and dispersion $\sigma_{\zeta}^{D}$ accounts for the intrinsic scatter about the DTF straight line $\Delta_{\mathrm{DTF}}$ of zero-point $b^{D}$ and slope $a^{D}$. The distance modulus $\mu$ of an object reads:

$\mu=m-M=5 \log _{10} r+25$

where $m$ is the apparent magnitude of the galaxy and $r$ its distance in Mpc. Regardless of measurement errors, the observed probability density takes the following form:

$\mathrm{d} P_{2}=\frac{1}{A_{2}} \phi(m, p) f_{p}(p) \mathrm{d} p g\left(\zeta^{D} ; 0, \sigma_{\zeta}^{D}\right) \mathrm{d} \zeta^{D} h(\mu) \mathrm{d} \mu$

where $\phi(m, p)$ is a selection function accounting for selection effects in observation on $m$ and $p, h(\mu)$ is the spatial distribution function of sources (along the line-of-sight) and $A_{2}=\int \phi(m, p) f_{p}(p) g\left(\zeta^{D} ; 0, \sigma_{\zeta}^{D}\right) h(\mu) \mathrm{d} p \mathrm{~d} \mu \mathrm{d} \zeta^{D}$ is the normalisation factor warranting $\int \mathrm{d} P_{2}=1$. Under the three following assumptions:

- $\mathcal{H} 0)$ No measurement errors on $m$ and $p$ are present. Particularly, corrections on galactic extinction and on inclination effects are supposed valid.

- $\mathcal{H} 1)$ The function $g\left(\zeta^{D} ; 0, \sigma_{\zeta}^{D}\right)$ is gaussian.
- H2) Galaxies are homogeneously distributed in space, which implies that, whatever the line-of-sight direction, the distance modulus distribution function reads $h(\mu)=\exp [3 \alpha \mu]$ with $\alpha=\frac{\ln 10}{5}$.

a statistical estimator $\tilde{r}$ generally adopted for the distance of a galaxy with measured $m$ and $p$ reads as follows (see Appendix A for details, or Lynden-Bell et al. 1988; Landy \& Szalay 1992; Triay et al. 1994):

$\tilde{r}=\exp \left[\alpha\left(m-a^{D} p-b^{D}-25\right)\right] \exp \left[\frac{7}{2} \alpha^{2} \sigma_{\zeta}^{D 2}\right]$

where the term $\exp \left[\frac{7}{2} \alpha^{2} \sigma_{\zeta}^{D 2}\right]$ accounts for a volume correction. This unbiased ${ }^{1}$ distance estimator does not depend on the selection function $\phi(m, p)$ in $m$ and $p$ and on the specific shape of the distribution function $f_{p}(p)$. Its accuracy $\Delta \tilde{r}$ is proportional to the distance estimate of the galaxy (see Appendix A):

$\Delta \tilde{r}=\tilde{r} \sqrt{\exp \left[\alpha^{2} \sigma_{\zeta}^{D 2}\right]-1}$.

The radial peculiar velocity $v$ of a galaxy, expressed in $\mathrm{km} \mathrm{s}^{-1}$ with respect to the velocity frame in which the redshift $z$ is measured, reads:

$v=z-H_{0} r$

where $H_{0}$ is the Hubble constant and $z$ is expressed in $\mathrm{km} \mathrm{s}^{-1}$ units. Assuming that the above hypotheses hold, the estimator $\tilde{v}$ of the radial peculiar velocity of a galaxy with measured $m, p$ and $z$ reads thus as follows:

$\tilde{v}=z-H_{0} \tilde{r}=z-B \exp \left[\alpha\left(m-a^{D} p\right)\right] \exp \left[\frac{7}{2} \alpha^{2} \sigma_{\zeta}^{D 2}\right]$

where $H_{0}$ and $b^{D}$ have been merged into a single parameter $B=H_{0} \exp \left[\alpha\left(-b^{D}-25\right)\right]$. The accuracy $\Delta \tilde{v}$ of this radial peculiar velocity estimator $\tilde{v}$ is:

$\Delta \tilde{v}=H_{0} \Delta \tilde{r}=H_{0} \tilde{r} \sqrt{\exp \left[\alpha^{2} \sigma_{\zeta}^{D 2}\right]-1}$.

\section{Calibration using null-correlation approach}

The presence of the radial peculiar velocities $v$ is included in the statistical modelization by rewriting the density probability $\mathrm{d} P_{2}$ of Eq. (5) as follows:

$\mathrm{d} P_{3}=\frac{1}{A_{3}} \phi(m, p) f_{p}(p) \mathrm{d} p g\left(\zeta^{D}\right) \mathrm{d} \zeta^{D} h(\mu) \mathrm{d} \mu f_{v}(v ; \mathbf{x}) \mathrm{d} v(11)$

1 The estimator $\tilde{r}$ given Eq. (6) is unbiased in the following sense. Suppose a sample of $N$ galaxies homogeneously distributed in space and with the same measured $m$ and $p$. For $N$ large enough, the distances average on the sample $\langle r\rangle$ will coincide with the estimator $\tilde{r}$ (i.e. $\langle r\rangle \rightarrow E(r \mid m, p)=\tilde{r}$ where $E(r \mid m, p)$ is the mathematical expectancy of $r$, given $m$ and $p$ ). The statistics of Eq. (6) is generally used for inferring the distance of individual galaxies. It amounts to apply the above statistical formalism on a sample containing only one object, which cannot be done without ambiguousness. 
where $f_{v}(v ; \mathbf{x})$ is the distribution function of radial peculiar velocities depending in general on the spatial position $\mathbf{x}=(r \cos l \cos b, r \sin l \cos b, r \sin b)$ with $(l, b)$ the direction of the line-of-sight in galactic coordinates.

The aim is herein to estimate the calibration parameters of the DTF relation entering into the radial peculiar velocity estimator $\tilde{v}$ of $\mathrm{Eq}(9)$ (i.e. the DTF slope $a^{D}$ and DTF "zero-point" defined as $\left.B^{*}=B \exp \left[\frac{7}{2} \alpha^{2} \sigma_{\zeta}^{D 2}\right]\right)$. The calibration sample is constituted of field galaxies selected along the same line-of-sight of direction $(l, b)$ for which apparent magnitude $m$, log line-width distance indicator $p$ and redshift $z$ are measured. It is assumed hereafter that the sample is described by the density probability of Eq. (11) and satisfies the following hypotheses:

- $\mathcal{H} 0$ ) After appropriate corrections (galactic extinction, inclination effects, ...), residual measurement errors can be neglected.

- $\mathcal{H} 1) g\left(\zeta^{D}\right)$ is gaussian, i.e. $g\left(\zeta^{D}\right)=g_{G}\left(\zeta^{D} ; 0, \sigma_{\zeta}^{D}\right)$.

- $\mathcal{H} 2)$ The distance modulus distribution function reads $h(\mu)=\exp [3 \alpha \mu]$ with $\alpha=\frac{\ln 10}{5}$.

- $\mathcal{H} 3)$ The distribution of radial peculiar velocities $v$ is a gaussian of mean $u$ and dispersion $\sigma_{v}$, i.e. $f_{v}(v ; \mathbf{x})=$ $g_{\mathrm{G}}\left(v ; u, \sigma_{v}\right)$.

Since the calibration sample is constituted of galaxies lying in the field, $\mathcal{H} 2$ appears as a reasonable assumption. Anyway, if the line-of-sight direction of the sample goes across a physical cluster, nothing prevents us to discard galaxies known to belong to the cluster.

Assumption $\mathcal{H} 3$ implies that the radial peculiar velocity field is not correlated with the distance $r . \mathcal{H} 3$ is thus ruled out if flows such like the Great Attractor are present along the line-of-sight. On the other hand, $\mathcal{H} 3$ is less restrictive than the pure Hubble flow hypothesis (i.e. $v=0$ everywhere). First, galaxies may have a Maxwellian agitation of velocity dispersion $\sigma_{v}$. Second, the mean radial peculiar velocity along the line-of-sight $u$ is not forced to zero. It allows to mimic the following situation. Suppose that a whole-sky sample is calibrated in a velocity frame of reference where sampled galaxies are not globally at rest (say that the sample has a bulk flow $\mathbf{u}=\left(u_{x}, u_{y}, u_{z}\right)$ in cartesian galactic coordinates with respect to the velocity frame of reference). The radial peculiar velocity of galaxies belonging to the same line-of-sight of direction $(l, b)$ will be shifted by $u=u_{x} \cos l \cos b+u_{y} \sin l \cos b+u_{z} \sin b$. Assumption $\mathcal{H} 3$ in fact tolerates this kind of situation.

\subsection{NCA calibration of the DTF slope $a^{D}$}

Calibration of the DTF slope $a^{D}$ using null-correlation approach (NCA) is based on the following remark. For a calibration sample satisfying assumptions $\mathcal{H} 0, \mathcal{H} 1, \mathcal{H} 2$ and $\mathcal{H} 3$ with $u=\sigma_{v}=0$ (i.e. pure Hubble flow hypothesis), the variable $X=X\left(a^{D}\right)$ defined as:

$X=X\left(a^{D}\right)=\alpha\left(m-a^{D} p\right)-\ln z$ is not correlated with $p$ (see Appendix $\mathrm{C}$ for proof):

If $u=\sigma_{v}=0$ then $\operatorname{Cov}\left(p, X\left(a^{D}\right)\right)=0$

where $\operatorname{Cov}(p, X)=E(p X)-E(p) E(X)$ is the covariance of variables $p$ and $X$. On the other hand, a wrong value of the DTF slope (i.e. $a_{F}=a^{D}+\Delta a$ ) correlates $p$ and the random variable $X\left(a_{F}\right)=\alpha\left(m-a_{F} p\right)-\ln z$ :

$\operatorname{Cov}\left(p, X\left(a_{F}\right)\right)=\Delta C_{0}=-\alpha \Delta a \operatorname{Cov}(p, p) \neq 0$

where $\operatorname{Cov}(p, p)=E\left([p-E(p)]^{2}\right)=\Sigma(p)^{2}$ is the square of the standard deviation of $p$. The null-correlation approach consists in adopting the value of the parameter $a$ verifying $\operatorname{Cov}(p, X(a))=0$ as the correct estimate of the DTF slope $a^{D}$ :

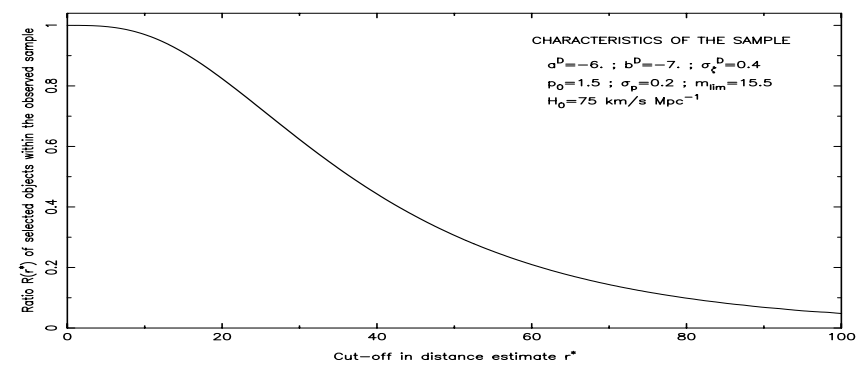

Fig. 1. Variation of the ratio $R\left(r^{*}\right)$ of selected object within the observed sample with respect to the extra cut-off in distance estimate $r^{*}$

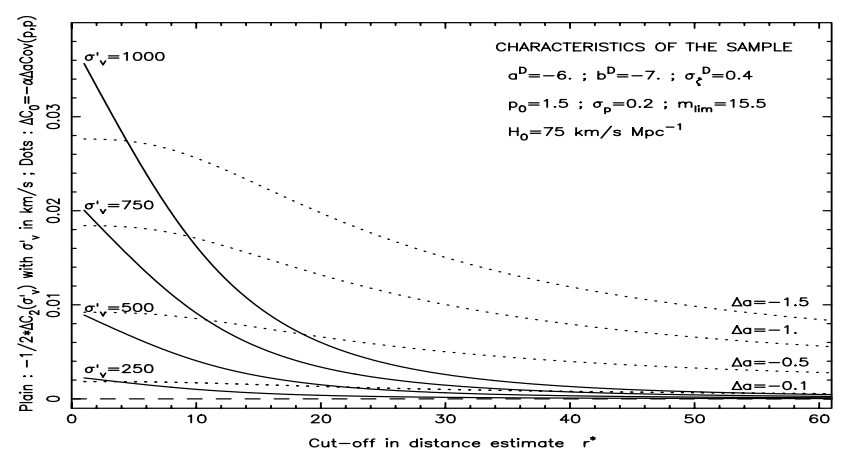

Fig. 2. Variation of the bias $\Delta C_{2}$ inferred by the presence of a Maxwellian velocity agitation of dispersion $\sigma_{\mathrm{v}}$ with respect to the extra cut-off in distance estimate $r^{*}\left(\sigma_{\mathrm{v}}^{\prime}=\left(\sigma_{\mathrm{v}}^{2}+u^{2}\right)^{1 / 2}\right)$

NCA estimate of $a^{D}: \quad a$ such $\operatorname{Cov}(p, X(a))=0$

which gives in practice:

$a^{\mathrm{NCA}}=\frac{\operatorname{Cov}(p, m)}{\operatorname{Cov}(p, p)}-\frac{1}{\alpha} \frac{\operatorname{Cov}(p, \ln z)}{\operatorname{Cov}(p, p)}$. 
Note that in the case of pure Hubble flow, the NCA is a quite robust technique for calibrating the slope of the DTF relation. It furnishes indeed an unbiased estimate of $a^{D}$ whatever the selection effects $\phi(m, p)$ on $m$ and $p$ which affect the observed sample, and whatever the specific shape of the theoretical distribution function $f_{p}(p)$ of the variable $p$ (see Appendix C).

Unfortunately, the presence of radial peculiar velocities such as the ones assumed in $\mathcal{H} 3$ with $u \neq 0$ or $\sigma_{v} \neq 0$ biases the NCA estimate of the slope $a^{D}$ since Eq. (13) rewrites in this case (see Appendix C):

$\operatorname{Cov}\left(p, X\left(a^{D}\right)\right)=-\operatorname{Cov}\left(p, \ln \left[1+\frac{v}{H_{0} r}\right]\right) \neq 0$.

However, the magnitude of this bias can be attenuated by selecting only distant galaxies of the observed sample. It is not surprising since the term $\ln \left[1+v /\left(H_{0} r\right)\right]$ in Eq. (17) becomes negligible for distances $r$ large enough. Such a subsampling can be performed by discarding galaxies of the observed sample which have a distance estimate $\tilde{r}$ smaller than a given $r^{*}$. It corresponds to introducing an extra selection function $\psi(m, p)$ defined as follows:

$\psi(m, p)=1$ if $\tilde{r} \geq r^{*} ; \psi(m, p)=0$ otherwise

where $\tilde{r}$ is given Eq. (6). Introducing this extra selection effect does not alterate the result obtained Eq. (13) since this property is insensitive to the specific shape adopted for the selection function on $m$ and $p$.

In order to evaluate amplitude of the bias appearing Eq. (17) and its variation with respect to the cut-off in distance estimate $r^{*}$, calculations have been performed on a synthetic sample characterized as follows:

- $\mathcal{H} 0, \mathcal{H} 1, \mathcal{H} 2$ and $\mathcal{H} 3$ are satisfied by the sample.

- DTF slope $a^{D}=-6$.

- DTF zero-point $b^{D}=-7$.

- DTF intrinsic scatter $\sigma_{\zeta}^{D}=0.4$.

- Hubble constant $H_{0}=75 \mathrm{~km} \mathrm{~s}^{-1} \mathrm{Mpc}^{-1}$.

- The distribution function of $p$ is a gaussian of mean $p_{0}=1.5$ and dispersion $\sigma_{\mathrm{p}}=0.2$.

- The selection effects in observation restrict to a cut-off in apparent magnitude $m_{\lim }=15.5$.

The two dominant terms of the bias created by the presence of radial peculiar velocities have been calculated (see Appendix C for details):

$$
\operatorname{Cov}\left(p, \ln \left[1+\frac{v}{H_{0} r}\right]\right)=\Delta C_{1}-\frac{1}{2} \Delta C_{2}+\circ\left(\Delta C_{2}\right)
$$

where the analytical expressions of $\Delta C_{1}$ and $\Delta C_{2}$ in function of $u, \sigma_{\mathrm{v}}$ and $r^{*}$ can be found Eq. (C11). The amplitude of these terms has to be compared with $\Delta C_{0}=$ $-\alpha \Delta a \operatorname{Cov}(p, p)$ appearing in Eq. (14) when a wrong value of the DTF slope is adopted. Expression of $\Delta C_{0}$ in function of $\Delta a$ and $r^{*}$ is given Eq. (C12).

Figure 1 shows variation of $R\left(r^{*}\right)$, i.e. the ratio of selected objects within the observed sample, with respect to the cut-off in distance estimate $r^{*}$ (analytical expression of $R\left(r^{*}\right)$ is given Eq. (C13)). On one hand, high values of $r^{*}$ are required in order to minimize as far as possible the amplitude of the bias created by radial peculiar velocities. On the other hand, accuracy $\sigma_{\mathrm{a}}$ of the NCA slope estimate depends on the size of the selected subsample (i.e. $\sigma_{\mathrm{a}} \propto R\left(r^{*}\right)^{-1 / 2}$ due to the intrinsic statistical fluctuations affecting the sample). Since theses two features are indeed competitive (i.e. $R\left(r^{*}\right)$ decreases when $r^{*}$ increases, compromise on the optimal value of $r^{*}$ has to be chosen with regard to the specific characteristics of the data sample under consideration.

The variations of term $\Delta C_{2}$, i.e. the contribution of a Maxwellian agitation of velocity dispersion $\sigma_{\mathrm{v}}$ to the bias of Eq. (19), are illustrated Fig. 2. Influence of $\Delta C_{2}$ on the NCA estimate of the DTF slope $a^{D}$ given Eq. (15) is obtained by comparing $\Delta C_{2}$ with the contribution of $\Delta C_{0}=-\alpha \Delta a \operatorname{Cov}(p, p)$ to the covariance of Eq. (14). For example, if the mean radial peculiar velocity along the line-of-sight $u$ is zero, the magnitude of the bias on the NCA estimate of $a^{D}$ created by a velocity field of dispersion $\sigma_{\mathrm{v}}=1000 \mathrm{~km} \mathrm{~s}^{-1}$ is greater than $\Delta a=-1.5$ for $r^{*}=0$, falls to $\Delta a=-1$. for $r^{*} \approx 10$ (i.e. $R\left(r^{*}\right) \approx 0.95$ ), equalizes $\Delta a=-0.5$ for $r^{*} \approx 20$ (i.e. $\left.R\left(r^{*}\right) \approx 0.8\right)$ and finally falls below $\Delta a=-0.1$ for $r^{*} \approx 60$ (i.e. $R\left(r^{*}\right) \approx 0.2$ ). Figure 2 reveals two important features.

- If nearby galaxies are not discarded from the observed sample, the presence of a Maxwellian velocity agitation for galaxies contaminates strongly the NCA estimate of the DTF slope $a^{D}$ (a velocity dispersion of $\sigma_{\mathrm{v}}=$ $500 \mathrm{~km} \mathrm{~s}^{-1}$ induces a bias on $a^{D}$ of magnitude $\Delta a \approx$ $-0.5)$.

- This bias can be rendered arbitrarily small by selecting only distant galaxies by means of the extra cut-off in distance estimate $r^{*}$.

Figure 3 shows the variations of $\Delta C_{1}$, i.e. the dominant term of the bias entering Eq. (19) due to the presence of a mean radial peculiar velocity along the line-of-sight $u$, with respect to $r^{*}$. A careful analysis of Fig. 3 leads to the three following remarks.

The term $\Delta C_{1}$ decreases a function of $r^{*}$ less rapidly as than $\Delta C_{2}$. For comparable values of $\Delta C_{1}$ and $\Delta C_{2}$ at $r^{*}=0$, say for $u=-750 \mathrm{~km} \mathrm{~s}^{-1}$ and $\sigma_{\mathrm{v}}^{\prime}=1000 \mathrm{~km} \mathrm{~s}^{-1}$, $\Delta C_{1}$ bias falls to $\Delta a \approx-1.5$ at $r^{*}=10$ (to be compared with $\Delta a \approx-1$. for $\Delta C_{2}$ bias), to $\Delta a \approx-1$. at $r^{*}=20$ $\left(\Delta a \approx-0.5\right.$ for $\left.\Delta C_{2}\right)$ and finally to $\Delta a \approx-0.5$ at $r^{*}=60$ $\left(\Delta a \approx-0.1\right.$ for $\left.\Delta C_{2}\right)$. It thus turns out that a particular attention has to be paid in priority to the presence of constant velocity fields.

As the existence of constant velocities strongly biases the NCA estimate of $a^{D}$ (at $r^{*}=0, \Delta a \approx-1.3$ for $u=-500 \mathrm{~km} \mathrm{~s}^{-1}$ ), discarding nearby galaxies by means of distance estimate selection appears as a quite crucial step. Note however that the situation is not so stringent for samples affected by bulk flow. Since the $\Delta C_{1}$ bias is antisymmetric with respect to $u$ (see Fig. 3), the bias on 


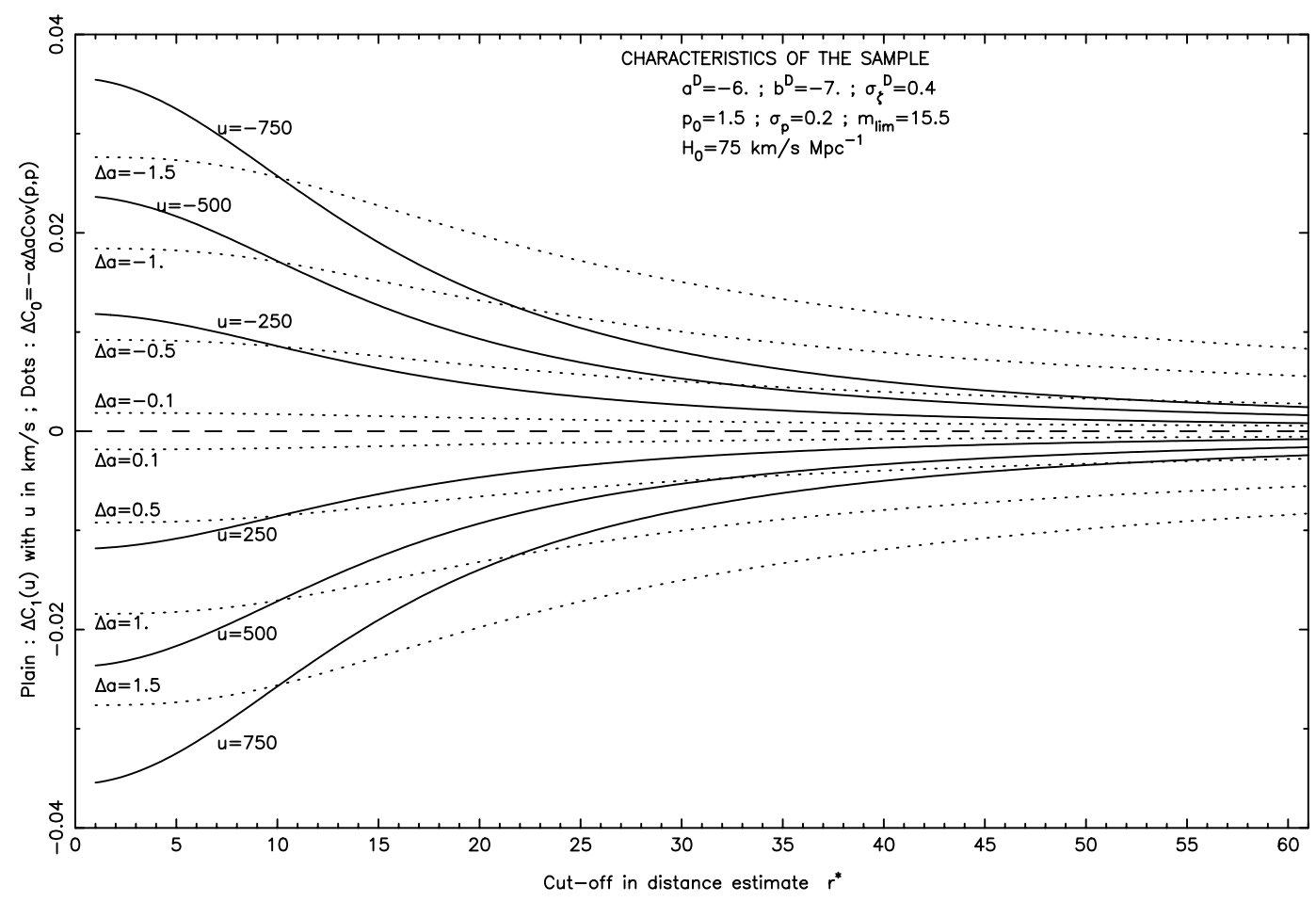

Fig. 3. Variation of the bias $\Delta C_{1}$ inferred by the presence of a constant velocity $u$ along the line-of-sight with respect to the extra cut-off in distance estimate $r^{*}$

the NCA estimate of $a^{D}$ cancels in average if the opposite line-of-sight direction is also considered. By scanning the sky by line-of-sight directions, this interesting symmetry allows indeed to detect bulk flows already at the level of the calibration step.

Finally, some upper bounds on the $a^{D}$ bias created by large-scale coherent velocity fields can be extracted from analysis of Fig. 3. Suppose that the line-of-sight of the calibration sample points toward the direction of a Great Attractor, located at a distance estimate of $\tilde{r}=40 \mathrm{Mpc}$ and creating back-side infall velocities, say of amplitude $u=-500 \mathrm{~km} \mathrm{~s}^{-1}$ at $\tilde{r}=50 \mathrm{Mpc}$ and slowly decreasing at larger distances. The bias on the NCA estimate of $a^{D}$ will be necessarily smaller than the $\Delta C_{1}$ bias for $u=$ $-500 \mathrm{~km} \mathrm{~s}^{-1}$ (i.e. at $r^{*}=50,|\Delta a|<0.5$ ). This property is closely related to the subsampling in distance estimate allowed by the null-correlation approach. The peculiar velocity field, whatever its specific form, becomes negligible compared to the mean Hubble flow as long as the cut-off in distance estimate $r^{*}$ is large enough. In this case the null-correlation approach furnishes unbiased estimate of the DTF slope $a^{D}$.

\subsection{NCA calibration of the DTF "zero-point" $B^{*}$}

Assuming that the DTF slope $a^{D}$ has been correctly calibrated by means of the technique presented in Sect. 3.1 or others calibration procedures, the null-correlation approach is herein used for calibrating the remaining calibration parameters entering into the radial peculiar velocity estimator $\tilde{v}$ of Eq. (9). For this purpose, this equation is rewritten as follows:

$\tilde{v}=z-H_{0} \tilde{r}=z-B^{*} \exp \left[\alpha\left(m-a^{D} p\right)\right]$

where $B^{*}=H_{0} \exp \left[\alpha\left(-b^{D}-25\right)\right] \exp \left[\frac{7}{2} \alpha^{2} \sigma_{\zeta}^{D 2}\right]$ depends on $H_{0}, b^{D}$ and $\sigma_{\zeta}^{D}$. For a calibration sample satisfying assumptions $\mathcal{H} 0, \mathcal{H} 1, \mathcal{H} 2$ and $\mathcal{H} 3$, the radial peculiar velocity estimator $\tilde{v}=\tilde{v}\left(a^{D}, B^{*}\right)$ is not correlated with $p$ (see Appendix B for proof):

$\operatorname{Cov}(p, \tilde{v})=\operatorname{Cov}\left(p, \tilde{v}\left(a^{D}, B^{*}\right)\right)=0$.

On the other hand, a wrong value of the $B^{*}$ parameter (i.e. $B_{\mathrm{F}}=B^{*}+\Delta B^{*}$ ) correlates $p$ and the random variable $\tilde{v}\left(a^{D}, B_{\mathrm{F}}\right)=z-B_{\mathrm{F}} \exp \left[\alpha\left(m-a^{D} p\right)\right]$ :

$\operatorname{Cov}\left(p, \tilde{v}\left(a^{D}, B_{\mathrm{F}}\right)\right)=-\frac{\Delta B^{*}}{B^{*}} \operatorname{Cov}\left(p, H_{0} \tilde{r}\right) \neq 0$

which does not vanish since selection effects on apparent magnitude $m$ correlate variables $p$ and $\tilde{r}$ (i.e. for selected galaxies, observable $p$ increases in average with the distance estimate $\tilde{r}$ ). Assuming that the DTF slope $a^{D}$ has been correctly calibrated, the NCA estimate of the "zeropoint" $B^{*}$ is then defined as:

NCA estimate of $B^{*}: \beta$ such $\operatorname{Cov}\left(p, \tilde{v}\left(a^{D}, \beta\right)\right)=0$ 
which gives in practice:

$B_{*}^{\mathrm{NCA}}=\frac{\operatorname{Cov}\left(p, \exp \left[\alpha\left(m-a^{D} p\right)\right]\right)}{\operatorname{Cov}(p, z)}$

NCA estimate of $B^{*}$ is clearly robust. It is insensitive to observational selection effects on $m$ and $p$, specific shape of the luminosity function $f_{p}(p)$, constant velocity field and Maxwellian agitation of galaxies (see Appendix B). Note however that presence of non-constant large scale velocity fields (such GA flow for example) biases NCA estimate of $B^{*}$. Unfortunatly the subsampling procedure in distance estimate previously proposed is not efficient for dealing with this kind of biases since the contribution of the peculiar velocity $v$ to $\operatorname{Cov}(p, z)$ entering Eq. (24) does not decrease with the distance $r$.

If one want to express the distance estimator $\tilde{r}$ of Eq. (6) in true distance units (Mpc), the Hubble constant $H_{0}$ has to be estimated (i.e. $\left.H_{0} \tilde{r}=B^{*} \exp \left[\alpha\left(m-a^{D} p\right)\right]\right)$. For this purpose, estimates of the parameters $B^{*}$-by using NCA calibration for example-, DTF zero-point $b^{D}$ - using primary distance indicators - and DTF intrinsic dispersion $\sigma_{\zeta}^{D}$-in galaxies clusters for example- are required (i.e. $\left.H_{0}=B^{*} \exp \left[\alpha\left(b^{D}+25\right)\right] \exp \left[-\frac{7}{2} \alpha^{2} \sigma_{\zeta}^{D 2}\right]\right)$. If $B^{*}$ is estimated with an error of $\delta B^{*}, b^{D}$ and $\sigma_{\zeta}^{D}$ with errors of $\delta b^{D}$ and $\delta \sigma_{\zeta}^{D}$ respectively, the dominant term of the relative error on the $H_{0}$ estimate reads:

$$
\frac{\delta H_{0}}{H_{0}} \approx \frac{\delta B^{*}}{B^{*}}+\alpha \delta b^{D}-7 \alpha^{2} \sigma_{\zeta}^{D} \delta \sigma_{\zeta}^{D} .
$$

\section{Conclusion}

A new technique for calibrating Tully-Fisher like relations was proposed. Based on a null-correlation approach, this calibration procedure is efficient when galaxies constituting the calibration sample are homogeneously distributed in space. The NCA technique is thus adequate for calibrating a sample of field galaxies for example.

In a first step was introduced the random variable $X(a)=\alpha(m-a p)-\ln z$ dependent on a slope parameter $a$ and on the observed apparent magnitude $m, \log$ line-width distance indicator $p$ and redshift $z$. In the case of pure Hubble flow (i.e. radial peculiar velocities are null everywhere), it was shown that variables $p$ and $X(a)$ are not correlated if and only if parameter $a$ equals the slope $a^{D}$ of the Direct TF relation. The NCA estimate of the DTF slope $a^{D}$ was defined as the value of $a$ such that correlation between $p$ and $X(a)$ vanishes. This estimator of $a^{D}$ was found particularly robust since it does not depend on the selection effects $\phi(m, p)$ on $m$ and $p$ which affect the observed calibration sample and on the specific shape of the luminosity function.

Influences of radial peculiar velocities was investigated in a second step. It was shown that the presence of a peculiar velocity field biases the NCA estimate of $a^{D}$. A procedure which consists in discarding nearby galaxies of the observed sample was introduced. Such subsampling is achieved by adding an extra selection function $\psi(m, p)$ in distance estimate $\tilde{r}$. The magnitude of the bias and its variations in function of the cut-off in distance estimate $r^{*}$ have been analysed on a synthetic sample and on the Mathewson spirals field galaxies sample (see Appendix D). The proposed subsampling procedure looks fairly efficient for minimizing bias on the NCA estimate of the DTF slope $a^{D}$ created by the presence of radial peculiar velocities.

In the third step, calibration of the "zero-point" parameter $B^{*}$ entering the definition of the velocity estimator $\tilde{v}$ was investigated. It was shown that the variables $p$ and $\tilde{v}\left(a^{D}, \beta\right)=z-\beta \exp \left[\alpha\left(m-a^{D} p\right)\right]$ are not correlated if and only if parameter $\beta$ equals the "zero-point" $B^{*}$. The NCA estimate of the second calibration parameter $B^{*}$ was thus defined as the value of $\beta$ such that correlation between $p$ and $\tilde{v}\left(a^{D}, \beta\right)$ vanishes. This $B^{*}$ estimator is robust. It is insensitive to observational selection effects on $m$ and $p$ and to the specific shape of the luminosity function. Moreover, NCA estimate of $B^{*}$ is not biased by the presence of a Maxwellian velocity agitation nor by the existence of bulk flow.

Acknowledgements. This work is one of the achievements of a long range program focusing on the statistical modelization of TF like relations, launched five years ago by Roland Triay. He is kindly thanked for constructive discussions. The hospitality of the Centre de Physique Théorique of Luminy is recognized. Stéphane Rauzy is cheerfully thanked for a generous financial support.

\section{References}

Aaronson M., Bothun G., Mould J., et al., 1986, ApJ 258, 64 Bertschinger E., Juszkiewicz R., 1988, ApJL 334, L59

Bertschinger E., Dekel A., Faber S.M., Burstein D., 1990, ApJ 364,370

Bicknell G.V., 1992, ApJ 399, 1

Bottinelli L., Gouguenheim L., Paturel G., Teerikorpi P., 1986, A\&A 156, 157

Dekel A., 1994, ARA\&A 32, 371

Ekholm T., 1996, A\&A 308, 7

Faber S.M., Jackson R., 1976, ApJ 204, 668

Fouqué P., Bottinelli L., Gouguenheim L., Paturel G., 1990, ApJ 349, 1

Hendry M.A., Simmons J.F.L., 1990, A\&A 237, 275

Hendry M.A., Simmons J.F.L., 1994, ApJ 435, 515

Hendry M.A., Rauzy S., Salucci P., Persic M., 1996, Astrophys. Lett. Comm. (in press) (astro-ph/9508138)

Landy S.D., Szalay A., 1992, ApJ 391, 494

Lynden-Bell D., Dressler A., Burstein D., et al., 1988, ApJ 326, 19

Mathewson D.S., Ford V.L., Buchhorn M., 1992, ApJS 81, 413

Newsam A.M., Simmons J.F.L., Hendry M.A., 1995, A\&A 294, 627 
Rauzy S., Lachièze-Rey M., Henriksen R.N., 1992, A\&A 256, 1

Rauzy S., Lachièze-Rey M., Henriksen R.N., 1995, Inverse Problems 11, 765 (astro-ph/9510017)

Rauzy S., Triay R., 1996, A\&A 307, 726

Sandage A., 1994, ApJ 430, 13

Schechter P.L., 1980, AJ 85, 801

Teerikorpi P., 1990, A\&A 234, 1
Triay R., Lachièze-Rey M., Rauzy S., 1994, A\&A 289, 19 Triay R., Rauzy S., Lachièze-Rey M., 1996, A\&A 309, 1 Tully R.B., Fisher J.R., 1977, A\&A 54, 661

Willick J.A., 1994, ApJS 92, 1

Willick J.A., Courteau S., Faber S.M., Burstein D., Dekel A., 1995, ApJ 446, 12

Willick J.A., Courteau S., Faber S.M., et al., 1996, ApJ 457, 460 